\title{
Basis for the Exercise-Induced Hyperphagia in Adult Hamsters
}

\author{
SARAH A. HOLDER BROWNE AND KATARINA TOMLJENOVIĆ BORER ${ }^{1}$ \\ Neuroscience Laboratory and Department of Physical Education, \\ University of Michigan, Ann Arbor, MI 48109
}

(Received 31 October 1977)

\begin{abstract}
BROWNE, S. A. H. AND K. T. BORER. Basis for the exercise-induced hyperphagia in adult hamsters. PHYSIOL. BEHAV. 20(5) 553-557, 1978. - In adult female golden hamsters (Mesocricetus auratus, Waterhouse) voluntary exercise is associated with increased food intake and accelerated somatic growth. We examined the possible interdependence between increased food intake and accelerated somatic growth by measuring food intake in hamsters whose growth was blocked by hypophysectomy. In a $2 \times 2$ design, hypophysectomized hamsters (HYPOX, $n=20$ ) and animals with intact pituitaries (INT, $n=24)$ were either allowed to exercise for 35 days $(n=22)$ or were maintained in sedentary condition $(n=22)$. Exercise induced increased food intake in both HYPOX and INT hamsters and somatic growth in INT hamsters only. Hypophysectomy was also associated with a $43 \%$ reduction in the activity level and a significant increase in the percentage of body fat estimated indirectly. We conclude that adult hamsters increase their food intake in response to some stimulus related to exercise and not to exercise-induced growth.
\end{abstract}

Exercise Food intake Hypophysectomy Growth Obesity

THE relationship between the energy expenditure in voluntary or forced exercise and the subsequent increase in food consumption is not well understood. In animals [13] and man [14] food intake is proportional to energy expended as work if sufficient time has been allowed for adjustment to regimes of vigorous physical activity. On the other hand, brief episodes of moderate physical activity are often associated with paradoxical decreases in food intake. For example, male rats decrease their food consumption during voluntary activity in rotating drums [8] or during forced exercise $[10,13,15,16,22,23]$. As a result, body weight is reduced primarily due to mobilization of body fat stores $[10,16]$. This has been interpreted as evidence that a threshold level of physical activity is necessary to bring sedentary body weights to a lower level characterized by greater sensitivity to the stimulus of energy expenditure [13]. A different formulation of a similar point of view is that voluntary exercise serves as a behavioral means of weight regulation in rodents $[8,24]$. Female rats, on the other hand, maintain stable body weights during forced exercise by virtue of increasing their food consumption [ 9 , $15,17]$. This sex difference in feeding and weight changes in response to exercise has been interpreted as evidence that female rat regulates its body weight more efficiently than the male rat [15].

An opportunity to examine the relationship between exercise and food intake presented itself following a recent report [1] of increases in food intake and weight gain stimulated by exercise in adult hamsters. A progressive increase in food intake was associated with voluntary exercise on horizontal activity discs. Hyperphagia persisted during the first week of retirement before food intake returned to control values. Increased weight gain was shown to reflect increased somatic growth rather than obesity. Exercise was associated with significant skeletal growth [7], with increases in the serum concentration of growth hormone [4], and with no consistent change in the percentage of body fat [6].

In our previous studies, increased activity, increased food intake and somatic growth tended to covary over the course of the experiment. Thus, increases in food intake may reflect either (1) a direct response to the increased caloric requirements of exercise, or (2) an indirect response to increased somatic growth. Preliminary observations in our laboratory indicated that hypophysectomized hamsters did not show typical patterns of somatic growth in response to exercise. We therefore examined the relative contributions of exercise and growth to increased food intake by comparing growth, exercise, and food intake in normal and hypophysectomized hamsters.

\section{METHOD}

\section{Animals}

Twenty-four female golden hamsters, Mesocricetus auratus, Waterhouse, over 100 days old and weighing about

\footnotetext{
${ }^{1}$ This research was supported in part by a grant from The Weight Watchers Foundation, Inc., to K. T. Borer. Requests for reprints should be sent to Dr. Katarina Borer, Department of Physical Education, University of Michigan, Ann Arbor, Michigan 48109.
} 
$120 \mathrm{~g}$ at the start of the experiment were obtained from Lakeview Farms (Newfield, NJ). Hypophysectomies were performed by Charles River Laboratories (Cambridge, MA) on 20 additional hamsters supplied by Lakeview Farms.

\section{Apparatus and Procedures}

Hamsters were individually housed in suspended wire cages at all times except during the 35-day period of exercise. During exercise hamsters were individually hou sed in Plexiglas boxes $30 \mathrm{~cm}$ wide, $40 \mathrm{~cm}$ deep, and $40 \mathrm{~cm}$ high, with a permanently mounted disc exerciser, $25 \mathrm{~cm}$ in dia. [1]. Animals were maintained in a room with controlled temperature $\left(22^{\circ} \mathrm{C}\right)$ and light-dark cycles (12L:12D).

A $2 \times 2$ factorial design was employed with variables of pituitary removal (HYPOX, $n=20$ ) or presence (INT, $n=$ $24)$ and of exercise (EX, $n=22$ ) or sedentary condition ( $\mathrm{SED}, \mathrm{n}=22$ ). Animals for the exercise and sedentary conditions were initially matched for body weight and body length. Each HYPOX group had 10 animals and INT groups had 12 animals each. Weights were taken daily and body lengths were measured on the first and on the last day of the experiment. Length measurements were taken from anesthetized hamsters (sodium pentobarbital IP, $75 \mathrm{mg} / \mathrm{kg}$ for INT and $50 \mathrm{mg} / \mathrm{kg}$ for HYPOX) by extending the animal's ventral surface over a cm scale.

Activity levels were recorded with the help of a microswitch and the electromagnetic counter as revolutions per day (RPD). Animals had unlimited access to Formulab Purina chow pellets which contain $3.51 \mathrm{Kcal} / \mathrm{g}$ and consist of $23 \%$ protein, $49.9 \%$ carbohydrate, and $6.5 \%$ fat. In addition, half of the animals within each of the four treatment groups were given unlimited access to sunflower seeds which contain $6.83 \mathrm{Kcal} / \mathrm{g}$ and have a composition of $23.5 \%$ protein, $9.7 \%$ carbohydrate, and $6.5 \%$ fat [1] with the objective of measuring the pattern of dietary selection. Since no change in dietary selection was found as a function of either exercise or pituitary manipulation, and dietary variation was evenly distributed across the four experimental groups, the data for the two dietary variables were combined.

Food intake was measured every $48 \mathrm{hr}$ taking into account any food spillage and is expressed as daily intake obtained by dividing the $48 \mathrm{hr}$ value by 2 . Food intake was expressed in $\mathrm{Kcal}$ per $100 \mathrm{~g}$ of hamster body weight to compensate for increases in body size seen in INT-EX group.

At the end of the experiment one half of HYPOX hamsters were subjected to $5 \mathrm{~min}$ of ether stress followed by cardiac puncture. This procedure facilitates growth hormone secretion in hamsters with intact pituitaries. Growth hormone values of HYPOX hamsters were extremely low and unaffected by the stress test [3]. In addition, sella turcica was examined for pituitary fragments in all of the HYPOX animals at the end of the experiment, and none were found. The percentage of body fat was determined indirectly from the total body water content which has a constant relationship of $73.2 \%$ to the lean body mass in the hamster [12] and other mammals [18]. Total body water content was determined by drying the carcasses to constant weight at temperatures which did not exceed $60^{\circ} \mathrm{C}$.

\section{Data Analysis}

Standard errors of the mean are used to express data variability. Differences between the four groups were evaluated by the $2 \times 2$ analysis of variance and the Scheffe simultaneous inference test. Mean food consumption during exercise and the first week of retirement was related to mean activity level for each exercising animal with least squares linear regression. Analysis of covariance was used to compare linear regressions of HYPOX and INT hamsters.

\section{RESULTS}

\section{Activity}

During the 35 days of exercise HYPOX hamsters ran on the average $22,165 \pm 2,500 \mathrm{RPD}$ while INT hamsters generated $39,069 \pm 3,419$ RPD. Hypophysectomy was therefore associated with a $43 \%$ reduction in voluntary running activity $(p<0.001)$.

\section{Food Intake}

Changes in food intake are presented in Fig. 1. Exercise was associated with an increase in food intake relative to sedentary controls beginning with the second and third weeks of exercise, respectively, in INT and HYPOX hamsters. Food intake remained high during the first week of retirement in HYPOX animals and during the initial two weeks of retirement in INT hamsters (Fig. 1). The overall mean daily food intake for the 35 days of exercise and the first week of retirement was $26.7 \pm 1.2 \mathrm{Kcal} / 100 \mathrm{~g}$ for HYPOX-EX hamsters, $22.8 \pm 0.9 \mathrm{Kcal} / 100 \mathrm{~g}$ for HYPOXSED hamsters, $34.0 \pm 0.7 \mathrm{Kcal} / 100 \mathrm{~g}$ for the INT-EX hamsters, and $24.6 \pm 0.7 \mathrm{Kcal} / 100 \mathrm{~g}$ for INT-SED hamsters. These values were significantly different due to the effects of pituitary variable $(F=27.3, p<0.0001)$, of activity variable $(F=57.1, p<0.0001)$, as well as of their interaction $(\mathrm{F}=9.7, p<0.003)$. In individual comparisons, food intake of HYPOX-EX hamsters was significantly greater than that of HYPOX-SED animals $(F=9.2$, $p<0.004)$ and significantly lower than that of INT-EX hamsters $(F=35.6, p<0.0001)$. INT-EX hamsters ate significantly more food than INT-SED hamsters $(F=61.2$, $p<0.0001)$, and HYPOX-SED hamsters ( $\mathrm{F}=83.4$, $p<0.0001)$. Sedentary animals with intact pituitaries ate the same amount of food daily as the HYPOX-SED hamsters.

A correlation between food intake and activity levels is presented in Fig. 2. The coefficient of correlation $r=0.765$ indicated that $58.5 \%$ of variability in food intake values was contributed by the variation in voluntary activity. The linear regression $y=0.00028 x+21.78$ and the food consumption values for sedentary animals, furthermore indicated that food intake first increased above the sedentary values when activity levels rose above 5,000 to 10,000 RPD.

In addition, the slopes of the regression of food intake and activity level were significantly different in HYPOX and INT hamsters $(p<0.03)$. The linear regression for HYPOX hamsters $(y=0.00037 x+18.228)$ showed a stronger correlation between food intake and activity $(\mathrm{r}=$ $0.790)$ than the corresponding linear regression for INT hamsters $(y=0.000094 x+30.065, r=0.430)$. Thus $62 \%$ of the variability in food intake was related to activity level in HYPOX hamsters while the corresponding value in INT animals was only $18.5 \%$. 


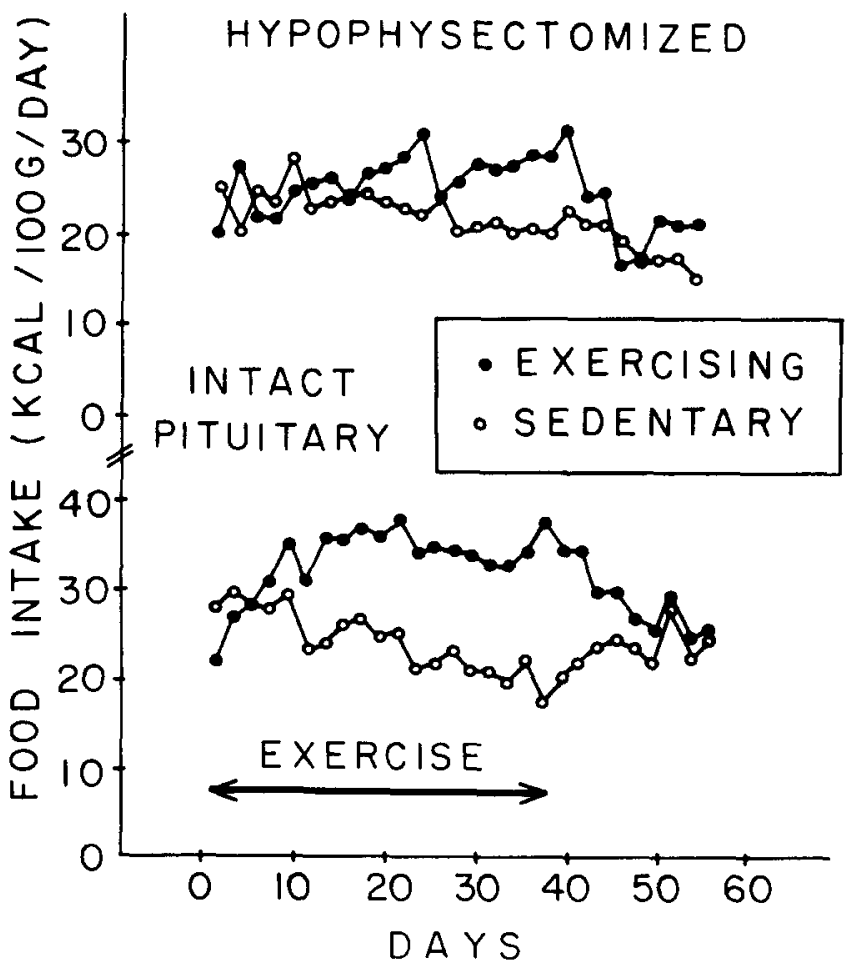

FIG. 1. Mean daily food intakes for exercising (solid circles) and sedentary (open circles) hypophysectomized hamsters (top) and hamsters with intact pituitaries (bottom).

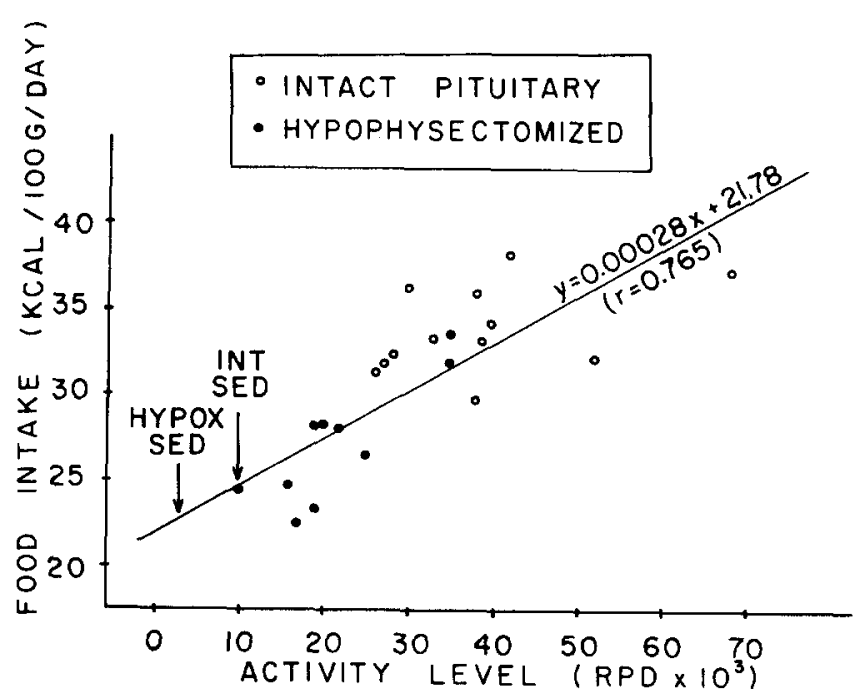

FIG. 2. Relationship between the mean food intake during the period of exercise and the first week of retirement and mean activity levels in hypophysectomized hamsters (solid circles) and hamsters with intact pituitaries (open circles).

\section{Somatic Changes}

Daily weight changes are shown in Fig. 3. Body weight of HYPOX-SED hamsters remained stable during the 10 weeks of the experiment, while body weights of INT-SED animals increased by about $10 \mathrm{~g}$ during the first three weeks

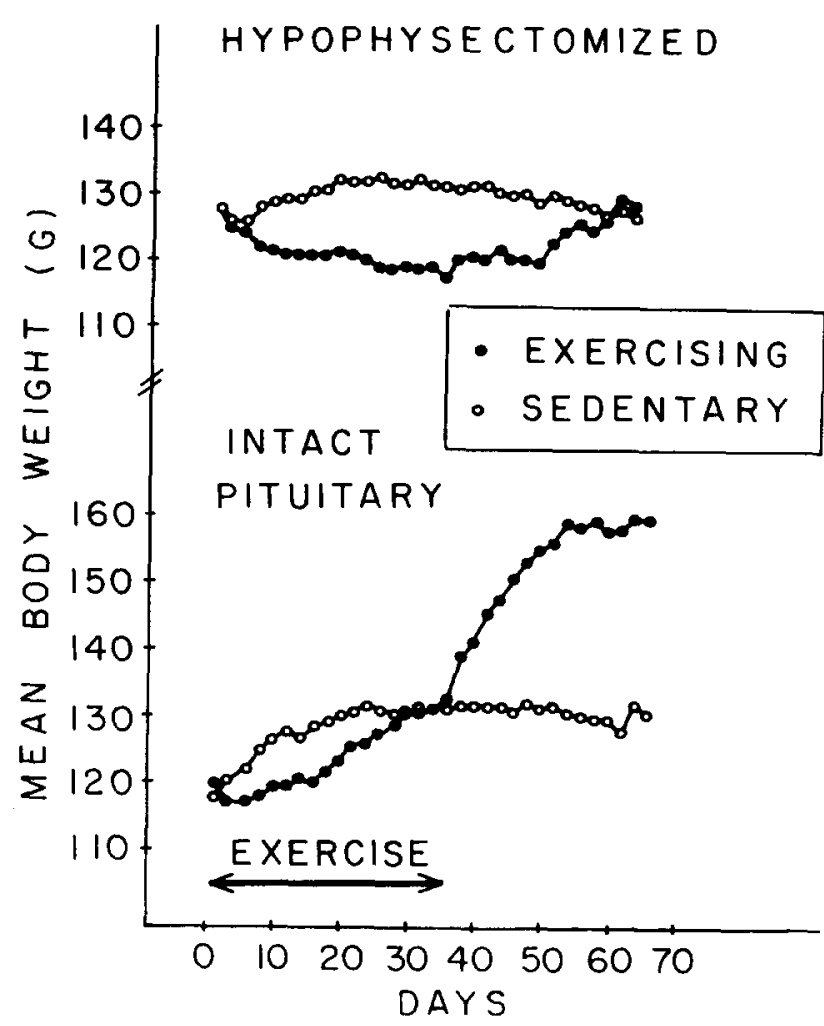

FIG. 3. Mean daily weight changes in exercising (solid circles) and sedentary (open circles) hypophysectomized hamsters (top) and hamsters with intact pituitaries (bottom).

of the experiment. Exercise affected the body weights of HYPOX and INT hamsters in different ways. HYPOX-EX hamsters displayed a steady weight loss throughout the 5 weeks of exercise and generated about $12 \mathrm{~g}$ of weight deficit. Following the retirement from discs, they gradually corrected this weight deficit. In contrast, INT-EX hamsters did not gain weight as rapidly as INT-SED hamsters during the first two weeks of the experiment and as a result their weight dropped about $7 \mathrm{~g}$ below the sedentary level. After that however, INT-EX hamsters gained weight at an increased pace for the remaining three weeks of exercise and during the three subsequent weeks of retirement. We used the weight change between the last day of the experiment and the first day of exercise to evaluate the effects of exercise in INT and HYPOX hamsters. Weight changes in the four groups were significantly different due to the effects of exercise $(F=10.3, p<0.002)$, of hypophysectomy $(\mathrm{F}=35.7, p<0.0001)$, and of the interaction between the two. HYPOX-EX and HYPOX-SED hamsters displayed similar weight changes $(0.23 \pm 3.9 \mathrm{~g}$ vs $-0.4 \pm 6.6 \mathrm{~g}$, respectively). Weight increments of INT-EX hamsters $(41.9 \pm 4.6 \mathrm{~g})$ were significantly greater than in the other 3 groups $(p<0.001)$. Weight increments of INT-SED hamsters $(12.9 \pm 2.9 \mathrm{~g})$ were significantly greater than in HYPOX-EX hamsters ( $p<0.05$, Fig. 3 ).

Table 1 presents changes in other somatic variables. Exercise had no effect on the percentage of body fat $(F=$ $0.35)$. Hypophysectomized hamsters, on the other hand appeared significantly fatter $(F=8.2, p<0.01)$ than the INT hamsters. INT groups grew significantly longer than 
TABLE 1

MEAN SOMATIC CHANGES

\begin{tabular}{lcccc}
\hline & \multicolumn{2}{c}{ Hypophysectomized } & \multicolumn{2}{c}{ Intact Pituitary } \\
& Exercising & Sedentary & Exercising & Sedentary \\
\hline & & & & \\
Percentage of & $19.6 \pm 2.7^{*}(10) \dagger \ddagger$ & $21.1 \pm 2.4(10) \ddagger$ & $16.2 \pm 1.4(6)$ & $9.4 \pm 0.5(5)$ \\
Body Fat & $0.9 \pm 1.0(10)$ & $2.8 \pm 1.4(10)$ & $9.3 \pm 1.4(12) \$ \uparrow$ & $5.3 \pm 1.3(12) \S$ \\
$\begin{array}{l}\text { Length Increment } \\
(\mathrm{mm})\end{array}$ & & & & \\
\hline
\end{tabular}

* Mean \pm SEM

†Numbers of animals are indicated within parentheses.

Pituitary variable: $\ddagger p<0.01, \$ p<0.001$

Activity $x$ pituitary variables: $\uparrow p<0.05$

the HYPOX groups ( $F=17.6, p<0.001)$. In addition, the increased growth in length stimulated by exercise in INT hamsters was disclosed in the significant interaction between pituitary and exercise variables $(F=5.1, p<0.01)$.

\section{DISCUSSION}

In the present study we have examined the question of why adult freely-exercising hamsters increase their food consumption during activity on horizontal discs and following the retirement from exercise. We have previously shown that hamsters with intact pituitaries display a striking elevation in food intake and in the rate of weight gain during and immediately following voluntary disc exercise $[1,6]$, and that this weight elevation represents acceleration of true somatic growth $[4,7]$. No studies to date have investigated whether food intake increased in response to exercise proper or whether it was secondarily related to exercise-induced acceleration of growth. To that end we measured food intake during exercise in adult hypophysectomized hamsters and in intact-pituitary hamsters. These groups differ radically in the growth response to exercise. We found that increases in food intake were directly related to exercise and independent of the exercise-induced acceleration of growth. Mean food consumption over the 5-week period of exercise and the first week of retirement was significantly higher in exercising hamsters regardless of whether their pituitary was removed or intact. Furthermore, the magnitude of the increase in food intake was systematically related to the level of physical activity in all hamsters regardless of pituitary variable. These findings suggest that the energy expenditure of different levels of activity exerts an influence over feeding controls. The mechanism by which exercise facilitates food intake is not known. The feeding mechanism responds to exercise with a latency of 7-14 days and remains activated for $7-10$ days after termination of exercise until the overall mean food consumption bears a direct relationship to the preceeding activity level (Fig. 2). From a linear regression relating food intake to the activity levels we determined that adult hamsters increase their food intake by about $2,74 \mathrm{Kcal} / 100 \mathrm{~g}$ body weight/day for each $1,000 \mathrm{RPD}$ increase in their activity level and independently of the presence or absence of a pituitary. When the sedentary food consumption is related to this regression, it appears that exercise first stimulates increases in food intake when activity levels exceed 5,000 to 10,000 RPD.
Our data suggest that exercise has a direct stimulatory effect over food intake in the hamster. In HYPOX animals incapable of rapid somatic growth, $62 \%$ of the variability in food intake was related to their activity level. In INT hamsters exercise also stimulated significant somatic growth (Table 1). Probably because of the additional dependent variable of growth, only $18.5 \%$ of the variability in the food intake of INT hamsters could be attributed to the variability in activity levels.

Stimulation of increased food intake and of increased growth may be initiated at different threshold activity levels. We have previously found that growth acceleration is associated with activity levels above 15,000 RPD [2]. We now report that food intake first increases when activity levels exceed 5,000 to 10,000 RPD.

Absence of weight increases in exercising HYPOX hamsters supports our previous conclusion $[1,2,6,7]$, that such weight changes represent somatic growth. Thus, exercise-induced weight increases depend on the presence of an intact pituitary. Lesions of rostral septal area lead to accelerated weight gain, skeletal growth, increased food intake, increased concentrations of growth hormone and insulin, and decreased pituitary content and concentration of growth hormone. All the somatic, endocrine, and behavioral effects of septal lesions in adult hamsters are blocked by hypophysectomy [5]. These two growth phenomena may be related. Septal lesions were shown to damage a portion of brain mechanism which maintains the slow asymptotic rate of growth characteristic of adult hamsters. Since exersice induces acceleration of somatic and skeletal growth $[1,6,7]$, accompanied by increases in the serum concentrations of growth hormone [4], and is ineffective in producing these somatic and endocrine changes after hypophysectomy (this study), it is probable that exercise leads to temporary obstruction of the same brain mechanism injured by septal lesions.

Two additional findings of the present study merit notice. First, hypophysectomy was associated with a $43 \%$ reduction in the voluntary activity levels. Maintenance of relatively high activity levels by hypophysectomized hamsters contrasts sharply with the complete cessation of running produced by hypophysectomy in rats [20]. This species difference seems to be related to difference in the dependence of voluntary activity on the activation by estrogens. Running activity in hypophysectomized rats is reinstated by administration of estradiol [19] but not of growth hormone $[20]$, just as the running activity in the rats of both sexes depends for its activation on estrogen or 
its derivatives $[21,24]$. In contrast, running activity in the hamster does not depend on the presence of intact gonads. Male animals run as vigorously as females [1] and gonadectomy does not affect activity levels in either sex (Borer, Bonnette, and Gibb, unpublished data).

Finally, hypophysectomized hamsters appeared to contain a significantly higher percentage of body fat regardless of whether they engaged in voluntary activity or remained sedentary (Table 1). Fat accumulation in hypophysectomized hamsters could reflect the loss of pituitary lipolytic hormones [11]. However, the decreased percentage of body water in HYPOX hamsters could also reflect other endocrine disturbances. Therefore our interpretation of increased obesity in HYPOX hamsters, based on the indirect estimates of body fat, should be validated by body fat measurements based on direct lipid-extraction methods.
In summary, we have determined that exercise stimulates increases in food intake in adult hamsters that are proportional to the levels of physical activity and independent of exercise-induced acceleration of growth. Hypophysectomized hamsters appeared to have a higher percentage of body fat and engaged in $43 \%$ less voluntary activity than the intact-pituitary hamsters. Our findings indicate that the effects of exercise over feeding controls are slow in onset, prolonged in duration, and quantitatively precise.

\section{ACKNOWLEDGEMENTS}

Richard Katz and Victor Katch made comments which helped improve the manuscript.

\section{REFERENCES}

1. Borer, K. T. Absence of weight regulation in exercising hamsters. Physiol. Behav. 12: 589-597, 1974.

2. Borer, K. T. and L. R. Kaplan. Exercise-induced growth in golden hamsters: Effects of age, weight, and activity level. Physiol. Behav. 18: 29-34, 1977.

3. Borer, K. T. and R. P. Kelch. A heterologous radioimmunoassay method for measurement of hamster growth hormone. Neuroendocrinology, 1978, in press.

4. Borer, K. T. and R. P. Kelch. Increased serum growth hormone and somatic growth in exercising adult hamsters. Am. J. Physiol. 1978, in press.

5. Borer, K. T., R. P. Kelch, M. P. White, L. Dolson and L. R. Kuhns. The role of septal area in the control of growth in adult hamsters. Neuroendocrinology 23: 133-150, 1977.

6. Borer, K. T. and A. A. Kooi. Regulatory defense of the exercise-induced weight elevation in hamsters. Behav. Biol. 13: $301-310,1975$.

7. Borer, K. T. and L. R. Kuhns. Radiographic evidence for acceleration of skeletal growth in adult hamsters by exercise. Growth 41: 1-13, 1977.

8. Collier, G. Work: a weak reinforcer. Trans. N. Y. Acad. Sci. 32: $557-576,1977$.

9. Crews, J. and E. A. Aldinger. Effect of chronic exercise on myocardial function. Am. Heart J. 74: 536-542, 1967.

10. Crews, E. L., III, K. W. Fuge, L. B. Oscai, J. O. Holloszy and R. E. Shank. Weight, food intake and body composition: Effects of exercise and of protein deficiency. Am. J. Physiol. 216: $359-363,1969$.

11. Kastin, A. J., T. W. Redding, R. Hall, G. M. Besser and A. V. Schally. Lipid mobilizing hormones of the hypothalamus and pituitary. Pharmac. Biochem. Behav. 3: 121-126, 1975.

12. Kodama, A. M. In vivo and in vitro determinations of body fat and body water in the hamster. J. appl. Physiol. 31: 218-222, 1971.

13. Mayer, J., N. B. Marshall, V. V. Vitale, J. H. Christensen, M. B. Mashayekhi and F. U. Stare. Exercise, food intake and body weight in normal rats and genetically obese adult mice. $A m$. J. Physiol. 177: 544-548, 1954.
14. Mayer, J., P. Roy and K. P. Mitra. Relation between caloric intake, body weight, and physical work: Studies in an industrial male population in West Bengal. Am. J. clin. Nutrit. 4: $169-175,1956$.

15. Nance, D. M., B. Bromley, J. R. Barnard and R. A. Gorski. Sexually dimorphic effects of forced exercise on food intake and body weight in the rat. Physiol. Behav. 19: 155-158, 1977.

16. Oscai, L. B. and J. O. Holloszy. Effects of weight changes produced by exercise, food restriction or overeating on body composition. J. clin. Invest. 48: 2124-2128, 1969.

17. Oscai, L. B., P. A. Mole and J. O. Holloszy. Effects of exercise on cardiac weight and mitochondria in male and female rats. Am. J. Physiol. 220: 1944-1948, 1974.

18. Pace, N. and E. N. Rathbun. Studies on body composition. III. The body water and chemically combined nitrogen content in relation to fat content. J. biol. Chem. 158: 685)691, 1945 .

19. Porterfield, A. L. and J. J. Stern. Growth hormone and the refractoriness of the prepubertal activity system to estradiol in the rat. Physiol. Psychol. 2: 23-25, 1974.

20. Richter, C. P. and G. B. Wisclocki. Anatomical and behavioral changes produced in the rat by complete and partial extirpation of the pituitary gland. Am. J. Physiol. 95: 481-492, 1930.

21. Roy, E. J. and G. N. Wade. Role of estrogens in androgeninduced spontaneous activity of female rats. J. comp. physiol. Psychol. 89: 573-579, 1975.

22. Stevenson, J. A. F., B. M. Box, V. Feleki and J. R. Beaton. Bouts of exercise and food intake in the rat. J. appl. Physiol. 21: $118-122,1966$.

23. Thomas, B. M. and A. T. Miller, Jr. Adaptation to forced exercise in the rat. Am. J. Physiol. 193: $350-354,1958$.

24. Wade, G. N. Sex hormones, regulatory behaviors and body weight. In: Advances in the Study of Behavior, edited by J. S Rosenblatt, R. A. Hinde, E. Shaw and C. C. Beer. New York: Academic Press, 1976. 